\title{
Diseño y puesta en funcionamiento de la "escalera musical" como medio saludable de relacionar áreas de conocimiento
}

\author{
Chao-Fernández, Rocío; Felpeto-Guerrero, Abraham²; Vázquez-Sánchez, Rubén ${ }^{3}$ \\ Universidade da Coruña. Grupo de investigación IMETIC, Facultad de Ciencias de la \\ Educación, Códigos ORCID: 10000-0002-6584-0049, 20000-0002-2943-5644, ${ }^{3} 0000-0002-$ \\ 0394-8951
}

\section{RESUMEN}

En la actualidad, en un intento de modernización de la escuela, las tecnologías de la información (TIC) forman parte habitual del proceso de enseñanza-aprendizaje. Sin embargo, según Díaz (2009, p.20), "no daremos por sentado que la utilización de las TIC es sinónimo de calidad educativa, ni mucho menos una garantía de aprendizaje" palabras que cobran especial relevancia en el caso de la enseñanza musical donde su uso se centra fundamentalmente en la edición de partituras, o edición y reproducción de audio.

Bajo este contexto surgió este proyecto, donde nuestro objetivo principal fue que el estudiantado pudiese comprobar y vivenciar la relación entre la altura musical y la altura geométrica. También pretendíamos evidenciar la relación de la música con otras áreas, como las matemáticas, la física y el arte, así como el fomento de estilos de vida saludables y la concienciación para evitar la contaminación acústica.

El proyecto, desarrollado por profesorado y alumnado de materias de música de Grado, Máster y Doctorado consistió en el diseño y puesta en funcionamiento de una instalación sonora en la Facultad de Ciencias de la Educación de la UDC, que transformaría las escaleras principales de la facultad en unas escaleras musicales con forma de piano.

PALABRAS CLAVE: Escalera musical; tecnología educative; instalación Sonora; uso didáctico de TIC's 


\section{CITA RECOMENDADA:}

Chao-Fernández, Rocío; Felpeto-Guerrero, Abraham; Vázquez-Sánchez, Rubén (2020): Diseño y puesta en funcionamiento de la "escalera musical" como medio saludable de relacionar áreas de conocimiento. En De la Torre Fernández, E. (ed.) (2020). Contextos universitarios transformadores: Boas prácticas no marco dos GID. IV Xornadas de Innovación Docente. Cufie. Universidade da Coruña. A Coruña (págs. 43-56).

DOI capítulo: https://doi.org/10.17979/spudc. 9788497497756.043

DOl libro: https://doi.org/10.17979/spudc. 9788497497756

\section{ABSTRACT}

Nowadays, in an attempt to modernize the school, information and communication technologies (ICT) are a regular part of the teaching-learning process. However, according to Díaz (2009, p.20), "we will not assume that the use of ICT is synonymous with educational quality, much less a guarantee of learning" words that are especially relevant in the case of music teaching where its use is mainly focused on editing music scores, or editing and reproduction of audio.

Under this context, this project emerged, where our main objective was that students could verify and experience the relationship between pitch (musical height) and geometric height. We also wanted to show the relationship of music with other areas, such as mathematics, physics and art, as well as the promotion of healthy lifestyles and awareness to avoid noise pollution.

The project, developed by teachers and students of music subjects of Undergraduate, Master and PhD studies consisted of the design and commissioning of a sound installation in the Faculty of Education Sciences of the UDC, which would transform the main stairs of the faculty on a piano-shaped musical stairs.

KEY WORDS: music stairs; educative technology; sound installation; didactic use of ICT 


\section{INTRODUCCIÓN}

Nos encontramos en un contexto educativo en el que cada vez resulta más habitual encontrar instrumentos y herramientas TIC dentro de los centros educativos. Sin embargo, contar con estos instrumentos no siempre está en consonancia con su uso, sino que depende en gran medida de la predisposición que tenga el profesorado hacia ellas, así como su opinión sobre su posible potencial didáctico (Domingo y Marqués, 2011) y en ocasiones hasta de factores como la propia edad del profesorado o el miedo a utilizarlas (Pavón, 2013).

En lo que atañe a la materia de música, bien es cierto que siempre ha sido una de las materias escolares susceptible de utilizar instrumentos tecnológicos dada su propia naturaleza y su necesidad de trabajar con algo intangible como es el sonido, sin embargo nos encontramos que aunque a lo largo de los años han ido apareciendo avances y nuevas herramientas TIC capaces de ser introducidas en el aula de música, su uso en la mayoría de los casos queda relegado a la grabación y reproducción musical bien por software o mediante dispositivos a tal efecto, a la transcripción y creación de partituras a través de software de edición y otras veces a la publicación de los resultados a través de blogs 0 webquests (Díaz, 2008).

Para tratar de dar una mayor visibilidad a las TIC dentro de la materia de música, mostrar diferentes posibilidades que ofrecen a mayores de las ya conocidas y mencionadas anteriormente, así como para poner de manifiesto la relación de la música con diferentes materias curriculares, desde el grupo de innovación metodológica a través de las TIC (IMETIC) se decide abordar el proyecto de creación y puesta en funcionamiento de la escalera musical, que aunque su misión principal era la de servir de recurso al alumnado para el aprendizaje y vivencia del concepto de altura musical en relación con la altura espacial, veremos que da juego para mucho más que eso, todo ello desde una vertiente lúdica y didáctica. 


\section{DESCRIPCIÓN DE LA EXPERIENCIA}

\subsection{LA ELECCIÓN DEL PROYECTO}

El primer reto al que nos enfrentábamos era el de elegir una temática lo suficientemente abierta como para que el proyecto pudiera abarcarse desde diferentes perspectivas. Además, debería ser lo más llamativo y atrayente posible para conseguir un efecto llamada de forma que las personas que lo vieran decidieran participar en el mismo, en lugar de ser meros espectadores. Haciendo retrospectiva sobre acciones realizadas en el pasado que utilizaran la música como reclamo y con gran poder de atracción, nos acordamos de una acción realizada en la estación de metro de Odenplan de la ciudad sueca de Estocolmo en el año 2009. En esta acción, desarrollada por Volkswagen bajo su concepto denominado "The fun theory", querían transmitir la idea de que hacer algo divertido es la forma más sencilla de producir un cambio positivo en el comportamiento de las personas (Combs, 2015).

Además, en relación con ese concepto de hacer algo divertido, la escalera musical sería el perfecto desencadenante para introducir uno de los términos que más están apareciendo en los congresos y publicaciones sobre innovación educativa en los últimos años, tal y como se ha podido ver en las propias Jornadas de Innovación Docente a las que va destinado este artículo. El término de gamificación en el aula. Extrapolando todo lo anterior, consideramos que tendríamos un proyecto divertido, que nos permitiría introducir el juego y además serviría para cambiar la conducta de las personas, pero esto no era suficiente.

\subsection{LA ELECCIÓN DE LOS CONCEPTOS A TRABAJAR DIDÁCTICAMENTE}

Una vez elegido el proyecto y conocidas sus características principales, existía un segundo paso: darle sentido educativo dentro de la materia de música. Para ello, debimos pensar qué concepto del currículo de educación musical era el más indicado para relacionar con unas escaleras, llegando a la conclusión de que la adquisición y comprensión del concepto de altura musical era el idóneo. Idóneo porque, aunque si bien cuando se estudia la notación musical 
pudiera quedar más o menos clara la relación espacial - en el pentagrama - grave-abajo, agudo-arriba, la realidad es que no es algo fácil de asociar por todos los niños dado que, entre otros, existen contradicciones sobre esta espacialidad si la extrapolamos a la interpretación de los diferentes instrumentos musicales, encontrándonos con que por ejemplo, en el piano tenemos grave-izquierda, agudo-derecha; en la guitarra o instrumentos de la familia de cuerda frotada tenemos que grave-arriba, agudo-abajo, entre otros muchos ejemplos (Burcet y Uzal, 2017). Por tanto, el proyecto de la escalera musical nos ayudaría enormemente a la adquisición de dicha relación conceptual-espacial.

Por otra parte, al mismo tiempo que se elegía el concepto principal a tratar, durante una sesión de lluvia de ideas emergieron muchos otros conceptos y áreas desde las que se podría abordar el uso didáctico de las escaleras musicales, lo que nos hizo abrir el proyecto ya no solo a la altura musical, sino a otros ámbitos que permiten relacionar la música de forma interdisciplinar con otras áreas de conocimiento. Se muestra a continuación alguna de estas relaciones.

- Música y matemáticas y/o geometría:

La primera relación es la anteriormente mencionada de altura musical ligada a la altura espacial, matemática o geométrica. Utilizando una escalera de 8 peldaños podemos asignar a cada uno de ellos una nota musical de forma que al ir subiendo por ellos físicamente por una parte vamos ganando al mismo tiempo altura en la escala musical y en la escala espacial (imagen 1).

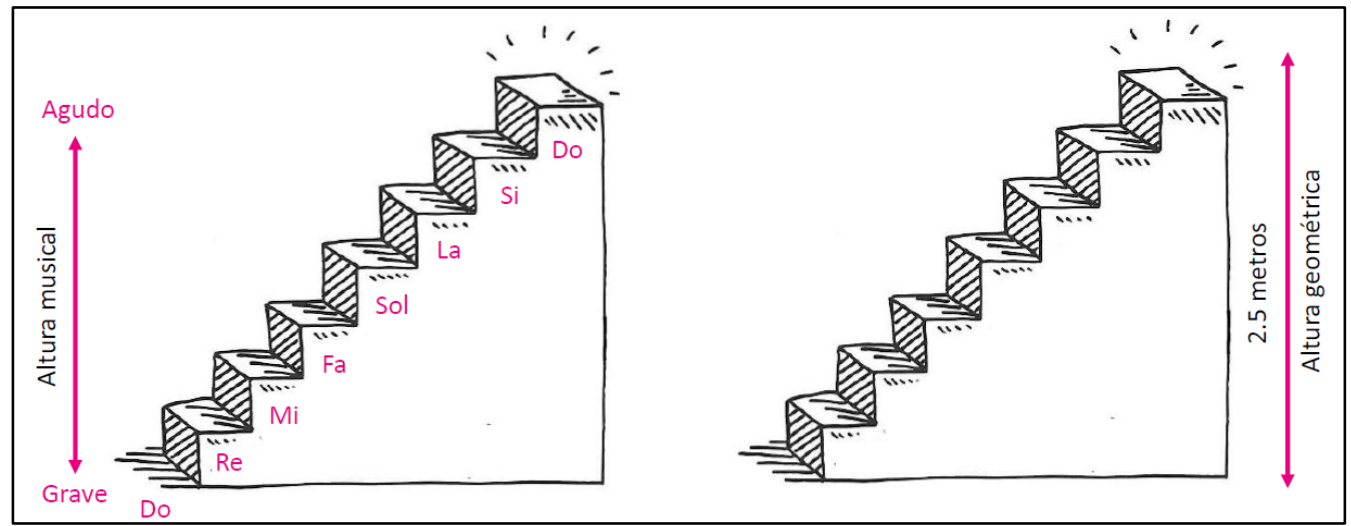

Imagen 1. Relación altura musical - altura espacial. Elaboración propia 
Una segunda relación matemática y/o geométrica es la que podemos encontrar en el concepto de intervalo musical. Musicalmente un intervalo no es más que la distancia - en altura - entre dos notas, pudiendo tener intervalos ascendentes o descendientes, dependiendo de la altura de la nota inicial y la final. Podemos hacer una analogía entre la distancia musical (que recorremos al ir de una nota a otra) y la altura espacial resultado de ir ascendiendo 0 descendiendo intervalos/peldaños en cada caso (imagen 2).

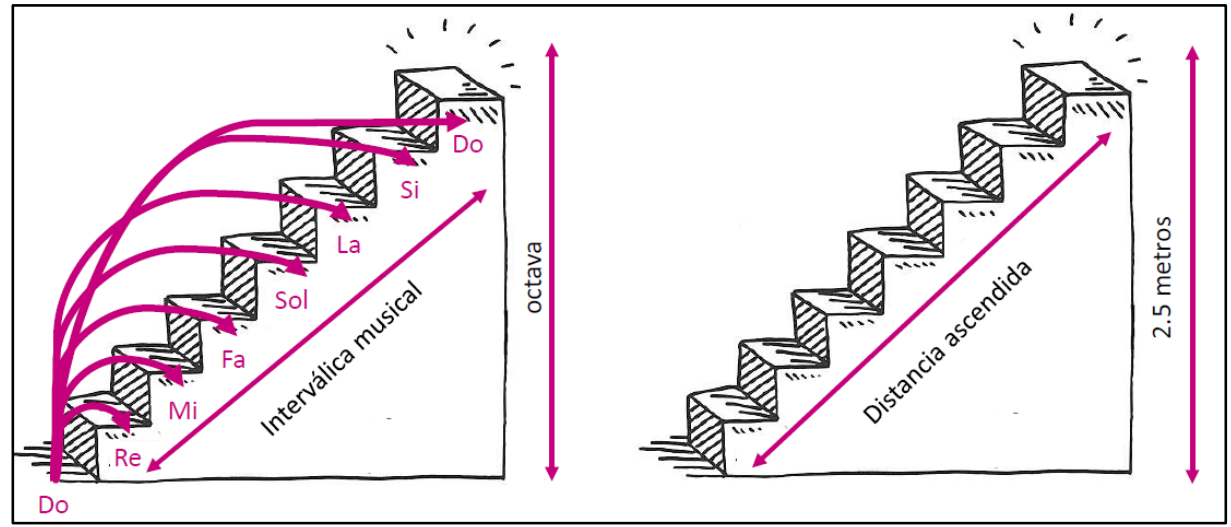

Imagen 2. Relación interválica musical - distancia ascendida. Elaboración propia

- Música y física:

Otro de los conceptos musicales que podemos trabajar y relacionar gracias a la escalera musical es el de intensidad sonora. La intensidad es una de las cuatro cualidades del sonido, que permite diferenciar un sonido suave de un sonido fuerte. Desde una escalera podemos demostrar fácilmente la relación entre intensidad sonora y su energía de caída si dejamos caer un objeto más o menos contundente desde diferentes alturas y comparamos los resultados. Cuanto mayor masa tenga el objeto y mayor velocidad adquiera durante su caída, más energía cinética tendrá dicho objeto en el momento de su impacto contra el suelo. Una vez llega el objeto al suelo, la onda sonora resultante - y por tanto su intensidad sonora - será proporcional a la energía que el objeto ha adquirido durante su caída (imagen 3). Por tanto, tendríamos que a mayor velocidad adquirida - podría relacionarse incluso con el concepto de la velocidad de ataque musical - mayor intensidad sonora producirá y viceversa. 


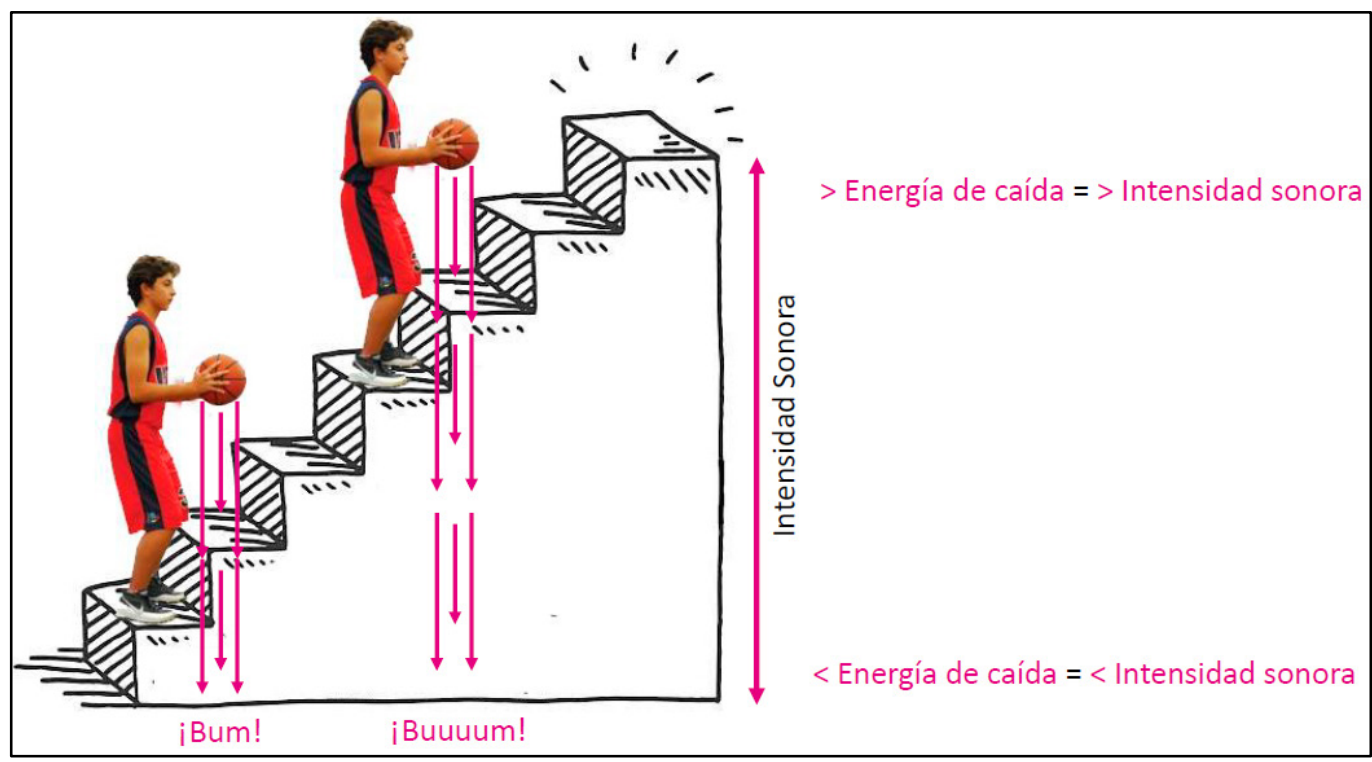

Imagen 3. Relación intensidad sonora - energía de caída. Elaboración propia

Por otra parte, como se verá más adelante en la sección que describe el funcionamiento de la escalera musical, podemos introducir el concepto de reflexión del sonido, fenómeno por el cual una onda sonora cuando se encuentra con un objeto bien se absorbe o bien cambia de dirección y se refleja. Como no podría ser de otra forma, la escalera musical funciona con sonido, de forma que son sensores de ultrasonidos colocados en cada peldaño los que se encargan de detectar mediante el fenómeno de la reflexión si algún objeto - en nuestro caso un pie - se interpone en el camino de la señal sonora emitida o incidente.

- Música y arte:

Resulta evidente el punto de vista clásico y la relación de la música con el concepto arte como parte integrante del mismo. Sin embargo, con la puesta en funcionamiento de la escalera musical, hemos querido sensibilizar a los visitantes hacia el arte desde el punto de vista contemporáneo en el que el espectador no actúa como mero observador inmóvil de la obra, sino que forma parte de la misma dándole identidad y sentido propios. La escalera musical no sería lo mismo sin participantes. No tendría sonido. $Y$ cada uno de dichos participantes 
actuará de forma diferente con la escalera, convirtiéndola así en algo vivo y único difícilmente repetible. Esto llevará al espectador a hacerle reflexionar sobre el concepto tradicional de arte y aprenderá a valorar e incluir las instalaciones sonoras como parte de dicho concepto.

- Música y otras disciplinas:

Lo mejor de todo es que no solo podemos relacionar conceptos musicales con otros conceptos de materias curriculares, sino que además con la puesta en funcionamiento de este proyecto estaremos fomentando entre otros la sostenibilidad, puesto que en el proyecto original se comprobó que el $66 \%$ de las personas dejaba de utilizar las escaleras mecánicas en beneficio de las escaleras manuales de toda la vida (Kim, 2015) y en nuestro caso aunque no lo cuantificamos, hemos notado que a pesar de la timidez del primer momento de la gente que pasaba por el lugar donde estaban las escaleras, después todo el mundo se animaba a usarlas en detrimento del ascensor. Además, al utilizar en este caso las escaleras musicales, estaremos acostumbrando a nuestro organismo a hacer actividad física - muchos de los participantes no dudaban en subir y bajar las escaleras en sucesivas ocasiones, algo impensable si no fueran musicales - y dicha actividad está demostrado que, si se realiza consecutivamente a lo largo del tiempo, beneficia en la reducción del índice de masa corporal y en la reducción del porcentaje de grasa (López, Marín y Castro, 2014), y además nos ayuda a reducir la huella de $\mathrm{CO}_{2}$ en el planeta y reducir la factura de la electricidad, ya que no estaremos utilizando el ascensor - en este caso - o las escaleras mecánicas. Por otra parte, también nos ha servido para crear conciencia sobre el medio ambiente sonoro, de forma que el volumen al que estaban funcionando las escaleras era bajo para que no resultara molesto ni repetitivo el sucesivo subir y bajar de la gente, pasando a formar parte del background noise (Truax, 1999) o ruido de fondo del edificio de la facultad mezclándose, camuflándose 0 enmascarándose con el resto de sonidos presentes. 


\subsection{EL FUNCIONAMIENTO DE LA ESCALERA MUSICAL}

Una vez teníamos claro para qué se iba a utilizar la escalera llegaba el momento de decidir cómo funcionaría. De las múltiples posibilidades - sensores láser, sensores de proximidad, sensores de presión, etc. - decidimos que una escalera musical no podría funcionar de otra forma sino con sonido, por tanto, el elemento que detectaría nuestro paso por cada escalón sería un sensor de ultrasonidos. El esquema de funcionamiento sería el siguiente:

Por una parte, necesitamos tanto sensores de ultrasonidos como peldaños de la escalera deseemos sonorizar, en nuestro caso se trataba de 8 peldaños y se decide hacer una escalera musical diatónica - sonidos Do, Re, Mi, Fa, Sol, La, Si, Do - por su mayor sencillez de montaje. Cada sensor iría conectado a una placa de control Arduino MEGA, que se encargaría de procesar la emisión y recepción de los pulsos sonoros así como energizar cada uno de los sensores de ultrasonidos, y dicho Arduino iría conectado a un ordenador que sería el que, con un programa informático creado ex profeso para la ocasión en el software de programación Processing, haría los cálculos necesarios y se encargaría de reproducir el sonido de cada peldaño de ser el caso en función de los datos recibidos de la placa Arduino.

Resumidamente, la secuencia de funcionamiento sería la que sigue: cada uno de los sensores de ultrasonidos colocados en el extremo de cada peldaño contiene un emisor y un receptor de ultrasonidos. El sensor está calibrado de forma que de cada 60 milisegundos envíe un pulso sonoro incidente. Dicho pulso incidente bien puede llegar al extremo contrario del escalón y rebotar en la pared creando un pulso reflejadas que llegará de vuelta al receptor de ultrasonidos 0 bien puede hacer lo mismo al encontrar algún obstáculo a lo largo de su camino. La placa Arduino registra el tiempo que tarda el pulso emitido en regresar al sensor. Estos datos son procesados internamente en Arduino para calcular la distancia que ha recorrido el pulso emitido de forma que, sabiendo cuál es la velocidad del sonido, con la fórmula Distancia $=($ tiempo/2) $\mathrm{x}$ velocidad del sonido. Una vez procesados los datos se envían al ordenador donde en el programa creado recibirá las distancias de detección de cada uno de los peldaños. Como de antemano sabemos la distancia que hay desde cada sensor hasta el extremo opuesto de cada 
peldaño, dado el caso de que la distancia calculada sea menor, eso significará que hay un obstáculo en medio del recorrido - un pie - y en ese momento el programa ordena reproducir el sonido asignado a cada peldaño hasta que se vuelva a obtener la distancia del recorrido completo, que nos indicaría que ya no está el obstáculo presente, y se el programa ordenaría la detención de la reproducción del sonido (imagen 4).

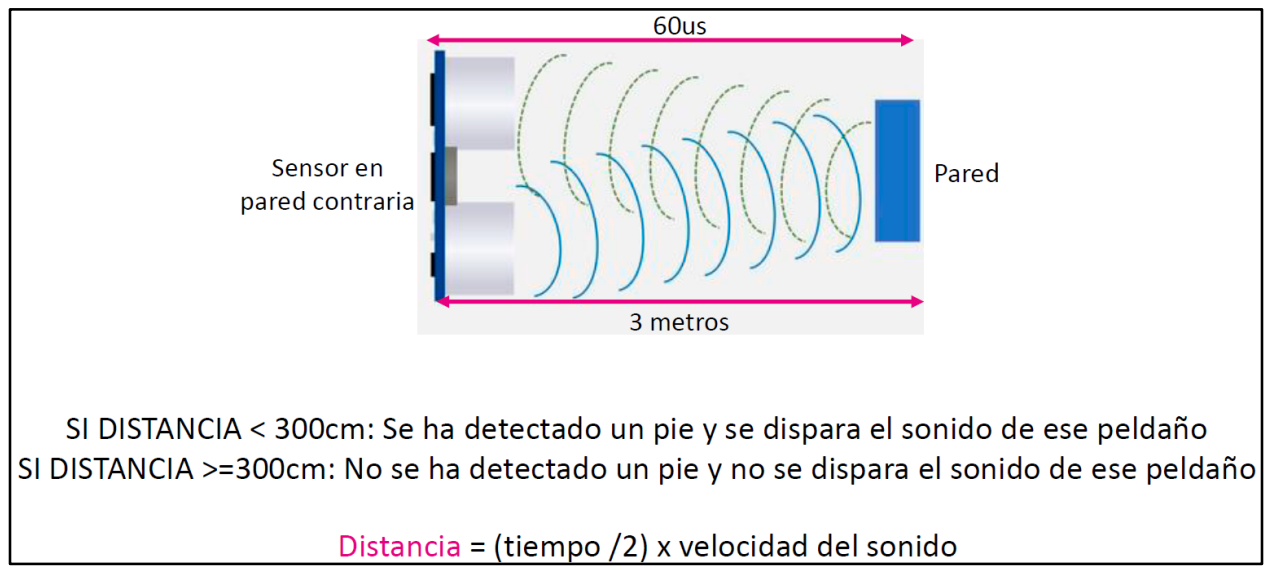

Imagen 4. Esquema de funcionamiento un sensor de ultrasonidos. Imagen modificada a partir de esquema de sensor SR-HCO4

\subsection{EL MONTAJE DE LA ESCALERA MUSICAL}

En último lugar, tras la planificación de todo el proyecto llegó el momento de realizar el montaje de la escalera musical, proceso ni mucho menos sencillo, dado que requería una serie de pasos previos a la instalación de la misma y además era necesario coordinar a un número elevado de participantes en un espacio reducido. En el montaje y creación colaboró el profesorado y alumnado de las materias de Pedagogía Musical, Investigación e Innovación en Educación Musical y Expresión Musical, así como de Nuevas Tecnologías: Estrategias didácticas. A mayores, coordinando y dirigiendo la experiencia, participaron dos estudiantes de doctorado del programa de Equidad e Innovación Educativa, dirigidos por una de las profesoras de las materias anteriormente citadas. El montaje se ha realizado en las siguientes fases: 
- Fase previa: donde se midieron las escaleras con el fin de hacer las estimaciones oportunas sobre la cantidad de material necesario.

- Preparación de vinilos: Una vez tomadas las medidas, se recortó vinilo de color blanco y negro para posteriormente forrar las escaleras para que su apariencia fuese la del teclado de un piano.

- Grabación de sonidos: Con la ayuda de una grabadora y varios instrumentos musicales - piano, flauta, clarinete, etc. - se grabaron una a una todas las notas que deberán sonar al pisar los escalones. Todas las grabaciones - 0 samples - deberían tener la misma duración. Posteriormente se limpiaron eliminando cualquier sonido no deseado que se pudiese interferir en la escucha.

- Preparación del cableado: Se hizo una colocación previa de todo el cableado a fin de comprobar si la longitud era correcta y para probar que funcionasen todas las conexiones, dejándolas así preparadas para el día de la instalación.

- Montaje final: El último día se pegaron los vinilos en las escaleras, se colocó el cableado con los sensores, el equipo informático y se hicieron las pruebas de funcionamiento para calibrar las escaleras (imagen 5).

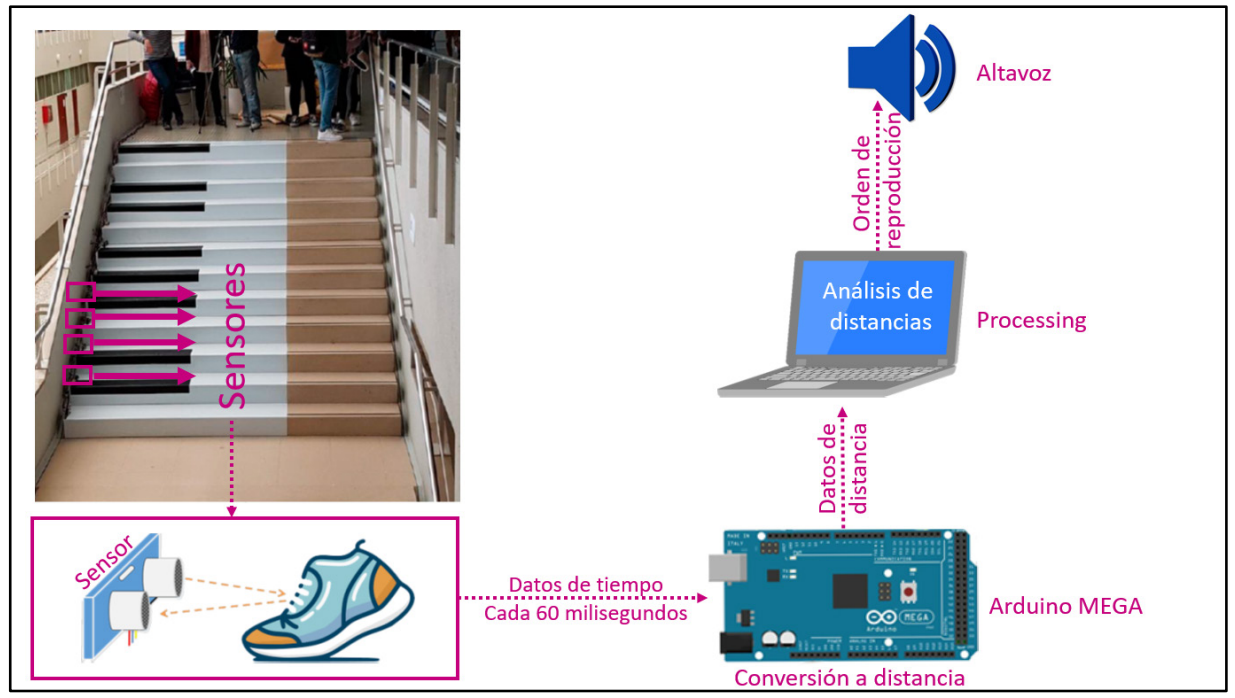

Imagen 5. Esquema del montaje final. Elaboración propia 


\section{RESULTADOS}

Durante los cuatro días que funcionó la escalera musical, logró un gran éxito y tuvo una excelente acogida dentro de la comunidad universitaria. Bien es cierto que durante los primeros momentos, las personas que se disponían a bajarlas eran un poco reticentes a utilizarlas porque al tratarse de una novedad y al haber más gente de lo habitual observando, producía en la comunidad universitaria un cierto grado de vergüenza y preferían utilizar el ascensor u otras escaleras, sin embargo, tras unos pocos minutos, al ir viendo que otras personas se animaban a participar y experimentar, tanto el ascensor como las escaleras secundarias pasaron a un segundo plano.

Se ha podido constatar que conceptos abstractos como la altura e interválica musical, que en ocasiones conllevan una gran dificultad en su aprendizaje, pueden llegar a comprenderse de forma sencilla utilizando la tecnología bajo una metodología inmersiva que haga partícipe al alumnado en la propia acción de aprendizaje y además permita relacionarla con otras áreas de conocimiento.

Al tratarse de una primera instalación de este tipo, no había total seguridad de que fuera a funcionar la escalera musical tal y como se había planeado, como así ha ocurrido, dado que se ha podido constatar el tipo de sensores utilizados no es el ideal para este tipo de proyecto, pudiendo comprobar que como efectivamente se pensaba, pueden afectar las condiciones de luz, humedad, e incluso los materiales de los que está hecho el calzado, en la propagación/captación de las ondas sonoras por parte de los sensores.

\section{CONCLUSIONES}

Tras esta experiencia podemos concluir que tal y como indicaban otras instalaciones similares, es más que observable un cambio de tendencia y conducta de las personas por el simple hecho de hacer que una actividad cotidiana como subir/bajar unas escaleras se convierta en un juego. De una forma sencilla se puede generar un mayor interés hacia el 
conocimiento y experimentación con conceptos no solamente musicales, sino de diversas áreas de conocimiento además de inculcar hábitos de vida saludables.

El uso de las TIC en educación musical no debe estar reñido a los usos generales, sino que el profesorado debe innovar buscando alternativas a los métodos clásicos de aprendizaje, merece la pena el riesgo y trabajo que ello conlleva, a la vista del interés que ha suscitado la puesta en práctica de esta experiencia ya no solamente en alumnado sino también en profesorado de diversas áreas.

\section{REFERENCIAS}

Bohyun, K. (2015). Gamification. Examples, Definitions and Related Concepts. Library Technology Reports, (51), pp. 10-16

Burcet, M. I., Uzal, S. (2018). La representación de la altura musical: un estudio con adultos en etapas iniciales de la adquisición de la notación musical. En N. Alessandroni y M.I. Burcet (Eds.), La experiencia musical. Investigación, interpretación y prácticas educativas. Actas del $13^{\circ}$ Encuentro de Ciencias Cognitivas de la Música (pp. 105115). Buenos Aires: SACCoM.

Combs, M. (2015). Musical Stairs: Encouraging Physical Activity through Persuasive Technology. Scholarly and Creative Work from DePauw University. Paper 24.

Díaz, G. (2008). Las TIC en el aula de música. En Pedrera, S. (Coord.), Percepción y expresión en la cultura musical básica, pp. 19-43. Madrid: Ministerio de Educación Cultura y Deporte, Subdirección General de Información y Publicaciones.

Domingo, M., Marquès, P. (2011). Aulas 2.0 y uso de las TIC en la práctica docente. Comunicar, XIX (37), pp.169-175.

López Á., Marín C. y Castro S. (2014). Efectos del uso de escaleras en la salud física. Revista Biosalud, 13(2), pp. 36-47 
Pavón, F. (2013). La introducción de las TIC en el currículum y en la organización escolar de la educación infantil y primaria. En Må. C. Martínez Serrano (Coord.), Buenas prácticas Educativas en el uso de las TIC. (pp.11-50). Jaén: Joxman

Truax, B. (1999). Handbook for acoustic ecology. Burnaby. Cambridge Street Records 